\title{
Continued seasonal circulation of enterovirus D68 in the Netherlands, 2011-2014
}

A Meijer (Adam.Meijer@rivm.nl)1, K S Benschop1, G A Donker², H G van der Avoort ${ }^{1}$

1. Centre for Infectious Disease Research, Diagnostics and Screening, National Institute for Public Health and the Environment (RIVM), Bilthoven, The Netherlands

2. NIVEL Primary Care Database, Sentinel Practices, Utrecht, The Netherlands

Citation style for this article:

Meijer A, Benschop KS, Donker GA, van der Avoort HG. Continued seasonal circulation of enterovirus D68 in the Netherlands, 2011-2014. Euro Surveill. 2014;19(42): pii=20935. Available online: http://www.eurosurveillance.org/ViewArticle.aspx?Articleld=20935

Article submitted on 17 October 2014 / published on 23 October 2014

Enterovirus D68 (EV-D68) continued to circulate in a seasonal pattern in the Netherlands, after the outbreak in 2010. Outpatient EV-D68 cases, mainly in the under 20 and 50-59 years age groups, presented with relatively mild respiratory disease. Hospital-based enterovirus surveillance identified more severe cases, mainly in children under 10 years of age. Dutch partial VP1 genomic region sequences from 2012 through 2014 were distributed over three sublineages similar to EV-D68 from the outbreak in the US in 2014.

After the 2010 outbreak, enterovirus D68 (EV-D68) continued to circulate in a seasonal pattern in the Netherlands. Here, we report the results of the monitoring of EV-D68 circulation in the Netherlands from week 12011 through week 402014 .

EV-D68 has been sporadically detected since its first description in 1962, up to 2008 [1,2]. From 2008 onwards, EV-D68 outbreaks occurred worldwide, including in 2010 in the Netherlands [2-5]. The largest outbreak is currently occurring in Northern America, causing substantial hospitalisation of children with severe respiratory disease in the United States (US) $[3,6]$. Many of these children have underlying disease, such as asthma [3,6]. Previous outbreaks described in the literature reported mainly on hospitalised patients [3].

In the Netherlands, retrospective analysis of enteroviruses detected from the general practitioner (GP) sentinel surveillance of influenza-like illness (ILI) and other acute respiratory infections (ARI) showed that circulation of EV-D68 occurred at least since 1996 up to the upsurge of 2010 [5]. EV-D68 cases had significantly more dyspnoea and bronchiolitis compared to EV-D68-negative patients with ILI or ARI notified in the same week [5]. In the Dutch national enterovirus surveillance aimed at exclusion of poliovirus circulation, EV-D68 was rarely detected, mainly because the focus has been on enteroviruses detected in stool specimens
[7]. Since 2010, we continued to monitor EV-D68 circulation in the Netherlands through both surveillance schemes.

\section{Specimen collection}

The methods used for specimen collection and for enterovirus detection and $\mathrm{VP}_{1}$ genomic region sequence analysis have been described $[5,7,8]$. For phylogenetic analysis using MEGA6 [9] all available VP1 sequences (covering nucleotides 132 through 471 relative to the $\mathrm{VP}_{1}$ gene of the Fermon strain) as of 12 October 2014 were downloaded from GenBank. The phylogeny was reconstructed using maximum likelihood and 1,000 bootstrap iterations with new Dutch sequences included (GenBank accession numbers KM975324-KM975350). Numbering of the major clusters (1, 2 and 3 ) has been described [5] and is synonymous to major clusters $B, C$ and $A$ respectively described by Tokarz et al. [10].

\section{Results}

Figure 1 and Table 1 summarise EV-D68 detections through the GP-based sentinel ILI and other ARI surveillance and the national enterovirus surveillance in the Netherlands, in specimens with collection dates from week 12011 through week 40 2014. Over the whole period, 27 EV-D68 cases were identified in a seasonal pattern; one in autumn 2011, 10 in autumnwinter period $2011 / 12$, five in autumn-winter period 2012/13, and 11 since summer 2014 (Figure 1). The start of detections in 2014 was earlier compared to the start of detections in 2012 (12 and six weeks earlier in the enterovirus and ILI/ARI surveillance respectively) and in 2013 (15 and 11 weeks earlier in the enterovirus and ILI/ARI surveillance respectively) (Figure 1). By year, the proportion EV-D68 among enteroviruses analysed was much higher (median 25\%; range 0-38\%) in the ILI/ARI surveillance compared to the enterovirus surveillance (median $0.5 \%$; range $0.3-1.4 \%$ (Table 1 ). However, by year, the percentage of enterovirus detections among ILI/ARI cases was low, on average $1.7 \%$ (range $1.4^{-}$ 2.1\%) (Table 1). 


\section{FIGURE 1}

Enterovirus D68 detections by source, the Netherlands, week 1 2011-week 402014

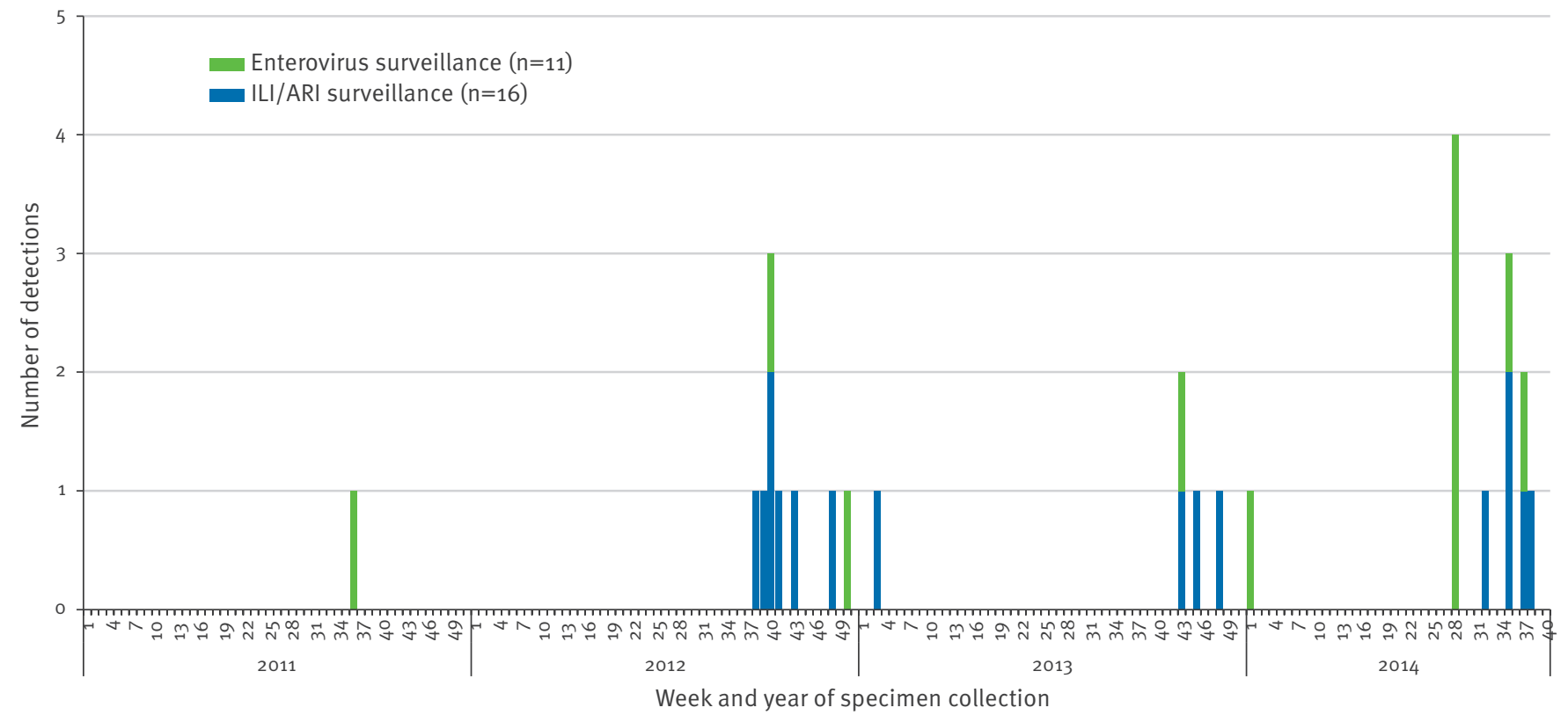

ARI: acute respiratory infections; ILI: influenza-like illness.

Due to increased awareness of the importance of enteroviruses in respiratory infections, laboratories participating in the Dutch national enterovirus surveillance also submitted enteroviruses associated with respiratory illness for typing after 2010; all 11 EV-D68 detections were in respiratory specimens. The age distribution in outpatients over the whole period was not different from that reported before, over the period 1996 through 2010 [5]; cases occurred mainly in the under 20 and in the 50-59 years age groups (Table 2). The male/female ratio was 1.3 (Table 2 ). In the national enterovirus surveillance, however, EV-D68 was mainly detected in the under 10 years age group and the male/ female ratio was 0.8 (Table 2 ).

The age distribution in 2014 was similar to that for the whole period for both surveillance schemes (data not shown). EV-D68 positive outpatients presented with ILI as well as other ARI, with most prominent symptoms being fever and cough (Table 2). Similar to the situation in Northern America in 2014, the hospitalised cases experienced severe respiratory disease (Table 2 ).

\section{TABLE 1}

Detections of enterovirus D68 in general practitioner sentinel influenza-like illness and other acute respiratory infection surveillance and in enterovirus surveillance, the Netherlands, week 1 2011-week 402014

\begin{tabular}{|c|c|c|c|}
\hline Year & $\begin{array}{c}\text { Number of clinical specimens } \\
\text { tested }\end{array}$ & $\begin{array}{c}\text { Number of enterovirus positive } \\
\text { specimens (\% of specimens } \\
\text { tested) })^{\mathrm{a}}\end{array}$ & $\begin{array}{c}\text { Number of enterovirus D68 } \\
\text { positive specimens (\% of } \\
\text { enterovirus positive specimens) }\end{array}$ \\
\hline \multicolumn{4}{|l|}{ ILI/ARI surveillance } \\
\hline 2011 & 1,369 & $19(1.4)$ & 0 \\
\hline 2012 & 1,126 & $24(2.1)$ & $7(29)$ \\
\hline 2013 & 1,292 & $19(1.5)$ & $4(21)$ \\
\hline \multicolumn{4}{|l|}{ Enterovirus surveillance } \\
\hline 2011 & Unknown & 362 & $1(0.3)$ \\
\hline 2012 & Unknown & 498 & $2(0.4)$ \\
\hline 2013 & Unknown & 309 & $2(0.6)$ \\
\hline 2014 (through week 40) & Unknown & 414 & $6(1.4)$ \\
\hline
\end{tabular}

ARI: acute respiratory infection; ILI: influenza-like illness.

a In enterovirus surveillance the number of enterovirus isolates or enterovirus positive clinical specimens submitted to the National Institute for Public Health and the Environment (RIVM) for VP1 typing is represented. 


\section{TABLE 2}

Demographic and clinical characteristics of enterovirus D68 positive patients from the general practitioner sentinel influenza-like illness and other acute respiratory infection surveillance and from the national enterovirus surveillance, the Netherlands, week 1 2011-week 402014

\begin{tabular}{|c|c|c|}
\hline Parameter & $\begin{array}{l}\text { ILI/ARI surveillance } \\
(\mathrm{N}=16) \\
\mathrm{n}\end{array}$ & $\begin{array}{c}\text { Enterovirus } \\
\text { surveillance }(\mathrm{N}=11) \\
\mathrm{n}\end{array}$ \\
\hline \multicolumn{3}{|l|}{ Age groups (years) } \\
\hline$<10$ & 5 & 8 \\
\hline $10-19$ & 3 & o \\
\hline $20-29$ & 1 & 2 \\
\hline $30-39$ & 0 & 0 \\
\hline $40-49$ & 1 & 0 \\
\hline $50-59$ & 4 & 1 \\
\hline $60-69$ & 1 & 0 \\
\hline $70-79$ & 1 & 0 \\
\hline$\geq 80$ & 0 & 0 \\
\hline \multicolumn{3}{|l|}{ Sex } \\
\hline Female & 7 & 6 \\
\hline Male & 9 & 5 \\
\hline \multicolumn{3}{|l|}{ Diagnosis $^{a}$} \\
\hline ILI & 8 & 0 \\
\hline Bronchitis & 4 & 2 \\
\hline Common cold & 3 & 0 \\
\hline Tonsillitis & 1 & 0 \\
\hline Pneumonia & 0 & 2 \\
\hline \multicolumn{3}{|l|}{ Symptoms ${ }^{a}$} \\
\hline Acute $^{b}$ & 14 & 0 \\
\hline Cough & 13 & 1 \\
\hline Fever & 13 & 0 \\
\hline Rhinorrhoea & 8 & 0 \\
\hline Sore throat & 8 & 0 \\
\hline Fatigue & 6 & 0 \\
\hline Headache & 5 & 0 \\
\hline Myalgia & 4 & 0 \\
\hline Dyspnoea & 4 & 2 \\
\hline Diarrhoea & 1 & 0 \\
\hline Underlying disease $^{c}$ & 5 & 2 \\
\hline $\begin{array}{l}\text { No clinical data } \\
\text { reported }\end{array}$ & 0 & 5 \\
\hline
\end{tabular}

ARI: acute respiratory infection; ILI: influenza-like illness.

a In ILI/ARI surveillance, diagnosis and symptoms are checkable items on the specimen form; in the enterovirus surveillance they are reported in a free text item.

b A prodromal stage of three or four days.

In ILI/ARI and enterovirus surveillance, underlying disease is reported in a free text item on the specimen form.

EV-D68 cases were detected all over the country; no localised outbreak was detected. Phylogenetic analysis of the $\mathrm{VP}_{1}$ genomic region showed that the Dutch EV-D68 from 2014, and from 2012 and 2013 as well, clustered with the US 2014 outbreak sequences in major group 1 in two sublineages and in major group 3 in one of the sublineages (Figure 2). Other Dutch EV-D68 from 2011 through 2014 clustered in other sublineages of major group 3 . The sequences in the two sublineages of major group 1 had highly similar amino acid signatures, whereas sequences in the sublineages of major group 3 had clearly different amino acid signatures, with most differences located in the immunogenic $B C$ and DE loops (Figure 3).

\section{Discussion}

Although rarely detected worldwide, our combined previous and current results over the period 1996-2014 show that EV-D68 seems to circulate every year in a seasonal pattern in the northern hemisphere in predominantly the autumn through early winter period, causing relatively mild respiratory illness in a number of individuals large enough to be picked up by the GP ILI/ARI surveillance [5]. The national enterovirus surveillance shows that a number of EV-D68 cases are admitted to hospital each year with more severe respiratory disease. Clinical presentation ranging from mild to severe respiratory disease is in line with our previous findings, and has been described before [1-6].

None of the patients described in this paper had symptoms of neurological disease or paralysis. A causative link between EV-D68 infection and paralysis has not been established to date [11]. Given the acute flaccid paralysis rate (AFP) indicator used by the World Health Organization for optimal polio surveillance (1-2 cases per 100,000 children below 15 years of age) one can expect that during a large EV-D68 outbreak, also several AFP patients will be shedding EV-D68. The present outbreak in Northern America provides an opportunity to investigate the link.

The difference in age distribution of EV-D68 cases between the ILI/ARI surveillance and the national enterovirus surveillance in our dataset is biased by the fact that $95 \%$ of enteroviruses identified by enterovirus surveillance are from children [7]. The male/female ratio of 1.3 among EV-D68 cases in the ILI/ARI surveillance was slightly lower compared to 1.5 over the period 1996 through 2010, but showing the usual male predominance among enterovirus infected persons [5]. Hence, the female predominance among EV-D68 cases in the national enterovirus surveillance is unusual, but likely the result of the low number of cases.

The number of hospitalised EV-D68 cases identified through the national enterovirus surveillance in the Netherlands is likely underestimated. When first described, EV-D68 was found to be relatively acid resistant and was distinguished from the acid-sensitive human rhinovirus type 87 (HRV87) on this basis [12]. However, in 2002, HRV87 was reclassified as EV-D68 based on phylogenetic analysis [13]. Many RT-PCR diagnostic tests for enteroviruses as well as rhinoviruses are targeted at the 5 ' untranslated region of the genome [8]. Many of these tests are capable of detecting EV-D68 despite mismatches in primers and probes with the EV-D68 target sites, although with varying sensitivity depending on reagents and equipment used for RT-PCR [8]. This might also result in a false negative or a false rhinovirus-positive result [8]. Performed 


\section{FIGURE 2}

Phylogenetic analysis of partial VP1 genomic region sequences of enterovirus D68, nucleotides 132 through 471 relative to the VP1 genomic region of the Fermon strain ${ }^{\mathrm{a}}$, covering the BC and DE immunogenic loops in the VP1 protein ${ }^{\mathrm{b}}$

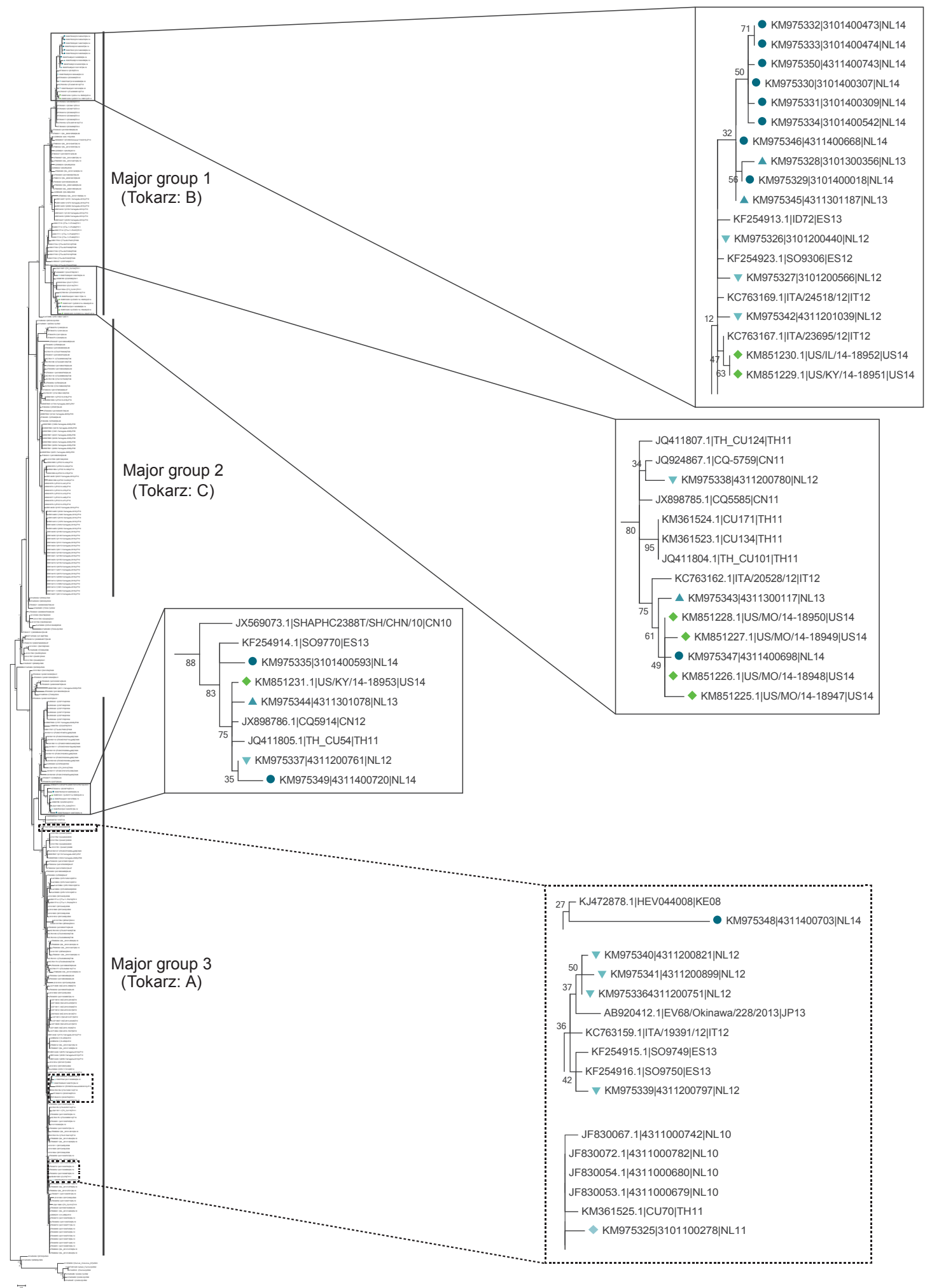

a GenBank ID: AFo81348.1.

b Figure 3.

c One enterovirus D68 from 2013 could only be identified by sequencing of the 5 ' untranslated region diagnostic RT-PCR product and is therefore not included in Figures 2 and 3.

The maximum likelihood tree is shown with the percentage bootstrap support for branching events after 1,000 iterations indicated at the nodes. Major phylogenetic groups as described in references 5 and 10 are indicated on the right of the tree.

Dutch sequences covering the period 2011-2014 and sequences

from the 2014 outbreak in the US are enlarged.

- Sequences from the US, 2014

Blue labels indicate sequences from the Netherlands:

$2011(n=1)$

$\checkmark 2012(n=9)$

$2013\left(n=4^{c}\right)$

- $2014(\mathrm{n}=12)$ 


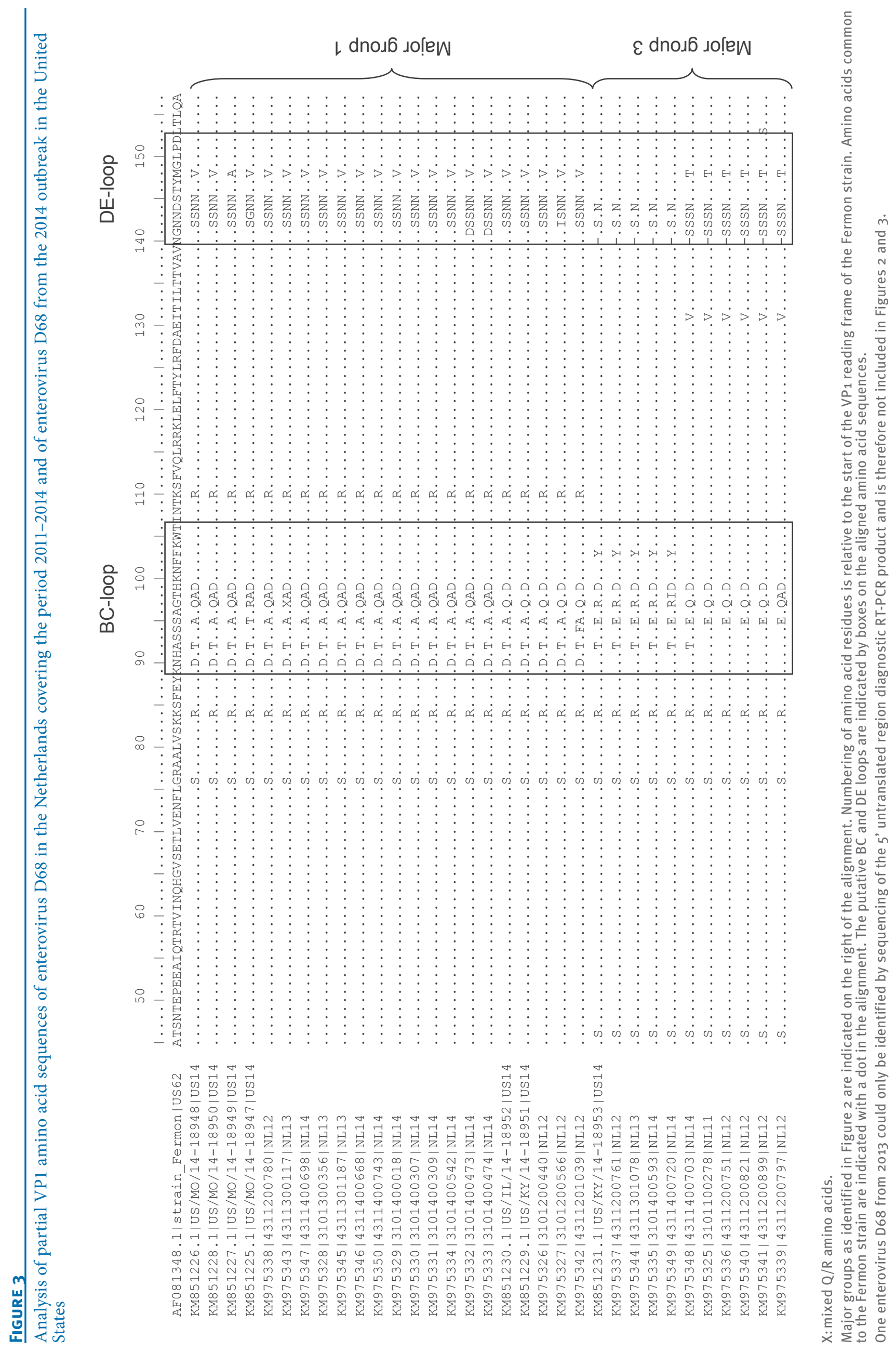


on respiratory specimens, these tests might therefore wrongly identify an EV-D68 virus as a rhinovirus, and further investigation by typing in the national enterovirus surveillance protocol will not be performed $[7,8]$. Furthermore, a number of Dutch laboratories have started to type enteroviruses themselves and share data through the national enterovirus surveillance, although this is done with delay and infrequently. These laboratories participate in VIRO-TypeNed (formerly called TYPENED) [14] to provide a year-round surveillance and current efforts are directed at updating VIRO-TypeNed with EV-D68 detections.

Previous work has indicated that co-circulation of the different phylogenetic lineages of EV-D68 is the result of increased variability of the $\mathrm{VP}_{1}$ genomic region, i.e. the $B C$ and DE loops, leading to reduced cross-neutralising antibodies raised against viruses of the major groups $[5,10,15]$. Variation of the highly conserved internal ribosome entry site in the 5 ' untranslated region, present in major group 1 and 2 viruses, has been suggested to be associated with increased virulence [10]. However, the US 2014 outbreak viruses are located in major groups 1 and 3, and similar viruses have been detected in the Netherlands, but associated with mild disease. Nevertheless, underlying disease like asthma seems to be an important factor for development of severe disease following EV-D68 infection [6]. Further in depth analysis of the EV-D68 full genomes from mild and severe cases and linked virological, clinical and epidemiological information should provide further insight in the factors determining severity of EV-D68 infection.

\section{Acknowledgements}

We thank Anne-Marie van den Brandt, Sharon van den Brink, Jeroen Cremer, Edin Jusic, Pieter Overduin, Bas van der Veer from the National Institute for Public Health and the Environment, Bilthoven, The Netherlands for laboratory support and Marianne Heshusius-van Valen from NIVEL Netherlands Institute for Health Services Research, Utrecht, the Netherlands for excellent assistance with data collection. We thank the participating Dutch clinical virological laboratories for submitting enteroviruses for typing, and general practitioners and their patients for participation in the ILI/ ARI sentinel surveillance. This work was partly funded by the Ministry of Health, Welfare and Sport, the Netherlands.

\section{Conflict of interest}

None declared.

\section{Authors' contributions}

Adam Meijer, Harrie van der Avoort and Kimberley Benschop collected data. Gé Donker coordinated the sentinel GP network collecting specimens. Adam Meijer performed the analysis of the data and wrote the first draft of the paper. All other authors reviewed the manuscript critically, and comments and suggestions were incorporated in the final version by Adam Meijer.
References

1. Khetsuriani N, Lamonte-Fowlkes A, Oberst S, Pallansch MA; Centers for Disease Control and Prevention. Enterovirus surveillance--United States, 1970-2005. MMWR Surveill Summ. 2006;55(8):1-20.

2. Centers for Disease Control and Prevention (CDC). Clusters of acute respiratory illness associated with human enterovirus 68--Asia, Europe, and United States, 2008-2010. MMWR Morb Mortal Wkly Rep. 2011;60(38):1301-4.

3. European Centre for Disease Prevention and Control (ECDC). Rapid Risk Assessment - Enterovirus 68 detections in the USA and Canada. Stockholm: ECDC. 26 Sep 2014. Available from: http://www.ecdc.europa.eu/en/publications/Publications/ enterovirus-68-USA-Canada-rapid-risk-assessment.pdf

4. Rahamat-Langendoen J, Riezebos-Brilman A, Borger R, van der Heide R, Brandenburg A, Schölvinck E, et al. Upsurge of human enterovirus 68 infections in patients with severe respiratory tract infections. J Clin Virol. 2011;52(2):103-6. http://dx.doi. org/10.1016/j.jcv.2011.06.019

5. Meijer A, van der Sanden S, Snijders BE, Jaramillo-Gutierrez G, Bont L, van der Ent CK, et al. Emergence and epidemic occurrence of enterovirus 68 respiratory infections in The Netherlands in 2010. Virology. 2012;423(1):49-57. http:// dx.doi.org/10.1016/j.virol.2011.11.021

6. Midgley CM, Jackson MA, Selvarangan R, Turabelidze G, Obringer E, Johnson D, et al. Severe respiratory illness associated with enterovirus D68 - Missouri and Illinois, 2014. MMWR Morb Mortal Wkly Rep. 2014;63(36):798-9.

7. van der Sanden SM, Koopmans MP, van der Avoort HG. Detection of human enteroviruses and parechoviruses as part of the national enterovirus surveillance in the Netherlands, 1996-2011. Eur J Clin Microbiol Infect Dis. 2013;32(12):1525-31. 10. Epub 2013 Jun 19.

8. Jaramillo-Gutierrez G, Benschop KS, Claas EC, de Jong AS van Loon AM, Pas SD, et al. September through October 2010 multi-centre study in the Netherlands examining laboratory ability to detect enterovirus 68, an emerging respiratory pathogen. J Virol Methods. 2013;190(1-2):53-62. http://dx.doi. org/10.1016/j.jviromet.2013.02.010

9. Tamura K, Stecher G, Peterson D, Filipski A, Kumar S. MEGA6: Molecular Evolutionary Genetics Analysis version 6.0. Mol Biol Evol. 2013;30(12):2725-9. http://dx.doi.org/10.1093/molbev/ mst197

10. Tokarz R, Firth C, Madhi SA, Howie SR, Wu W, Sall AA, et al. Worldwide emergence of multiple clades of enterovirus 68. Gen Virol. 2012;93(Pt 9):1952-8. http://dx.doi.org/10.1099/ vir.0.043935-0

11. Pastula DM, Aliabadi N, Haynes AK, Messacar K, Schreiner T, Maloney J, et al. Acute neurologic illness of unknown etiology in children - Colorado, August-September 2014. MMWR Morb Mortal Wkly Rep. 2014;63(40):901-2

12. Schieble JH, Fox VL, Lennette EH. A probable new human picornavirus associated with respiratory diseases. Am J Epidemiol. 1967;85(2):297-310.

13. Ishiko H, Miura R, Shimada Y, Hayashi A, Nakajima H, Yamazaki $\mathrm{S}$, et al. Human rhinovirus 87 identified as human enterovirus 68 by VP4-based molecular diagnosis. Intervirology. 2002;45(3):136-41. http://dx.doi.org/10.1159/000065866

14. Niesters HG, Rossen JW, van der Avoort H, Baas D, Benschop $\mathrm{K}$, Claas EC, et al. Laboratory-based surveillance in the molecular era: the TYPENED model, a joint data-sharing platform for clinical and public health laboratories. Euro Surveill. 2013;18(4):20387. Available from: http://www. eurosurveillance.org/ViewArticle.aspx?Articleld=20387

15. Imamura T, Okamoto M, Nakakita S, Suzuki A, Saito M Tamaki R, et al. Antigenic and receptor binding properties of enterovirus 68. J Virol. 2014;88(5):2374-84. Epub 2013 Dec 26. http://dx.doi.org/10.1128/JVI.03070-13 